\title{
ANDAMIAJE Y EVALUACIÓN FORMATIVA: DOS CARAS DE LA MISMA
} MONEDA

Scaffolding and formative assessment: two sides of the same coin

Mentoria e avaliação formativa: duas faces da mesma moeda

Ángel Pérez-Pueyo (1)

David Hortigüela Alcalá (2)

Carlos Gutiérrez-García (3)

Alejandra Hernando Garijo (4)

(1) Universidad de León, España. Teléfono: +34 987293024. Correo electrónico: angel.perez.pueyo@,unileon.es

(2) Universidad de Burgos, España. Teléfono: +34 947259517. Correo electrónico: dhortiguela@ubu.es

(3) Universidad de León, España. Teléfono: +34 987293058. Correo electrónico: cgutg@unileon.es

(4) Universidad de Burgos, España. Teléfono: +34 947259517. Correo electrónico: ahgarijo@ubu.es

\section{Resumen}

La evaluación formativa es una poderosa herramienta de aprendizaje. Cuando se vincula a procesos de andamiaje, puede generar un mayor nivel de implicación de los estudiantes y promover la autorregulación de su aprendizaje. Este taller presenta, de forma detallada, el proceso de andamiaje y evaluación formativa llevado a cabo en una asignatura de grado universitario. Asimismo, se identificarán situaciones óptimas para implementar procesos triádicos de evaluación compartida, que integren de forma sólida y coherente autoevaluación, coevaluación y heteroevaluación.

Palabras clave: Andamiaje; evaluación formativa; evaluación triádica; instrumentos de evaluación

\begin{abstract}
Formative evaluation is a powerful learning tool. When linked to scaffolding processes, it can improve students' involvement and promote self-regulation of their learning. This workshop presents, in a detailed way, the process of scaffolding and formative assessment carried out in a university degree course. Likewise, optimal situations to implement triadic shared assessment processes, which integrate in a solid and coherent way self-evaluation, co-evaluation and hetero-evaluation, will be identified.
\end{abstract}


Palavras-chave: Scaffolding; formative assessment; triadic assessment; assessment tools

\section{Resumo}

A avaliação formativa é uma poderosa ferramenta de aprendizagem. Quando aliada a processos de mentoria, pode gerar um maior envolvimento dos alunos e promover a auto-regulação da sua aprendizagem. Este workshop apresenta, de forma detalhada, o processo de mentoriae avaliação formativa realizados num curso do ensino universitário. Da mesma forma, serão identificadas situações ótimas para implementar processos triádicos de avaliação partilhada, que integram de forma sólida e coerente a autoavaliação, a coavaliação e a heteroavaliação.

Keywords: Mentoria; avaliação formativa; avaliação triádica; instrumentos de avaliação

\section{Andamiaje y evaluación formativa}

La evaluación formativa se ha convertido en uno de los referentes fundamentales para el desarrollo y mejora de procesos de aprendizaje eficaces con el alumnado (Biggs, 2005; Black \& Williams, 1998; Boud, 1995; Boud \& Falchikov, 2007; Dochy, Segers \& Dierick, 2002; López-Pastor, 1999, 2006, 2013; López-Pastor \& Pérez-Pueyo, 2017). Según Condemarín y Medina (2000), la evaluación formativa es un proceso que realimenta el aprendizaje y posibilita su autorregulación por parte del estudiante. En este sentido, Richard y Godbout (2000) defienden su realización como parte integral del proceso de enseñanza-aprendizaje en educación. A través de su utilización, se proporcionan feedbacks de manera regular con la intención de generar mejoras en el aprendizaje e implicar al alumnado en procesos de autoevaluación, coevaluación y evaluación compartida. Estos, a su vez, implican procesos de metacognición y reflexión, en los que se pretende utilizar los conocimientos de manera creativa para resolver problemas reales, transferibles a la vida cotidiana (Villardón, 2006).

Con todo, este entretejido de tareas de evaluación formativa se acaba dirigiendo también hacia un evento o resultado sumativo, por lo que a esta organización podría denominarse "andamiaje" (Moreno-Olivos, 2007). El concepto de andamiaje (scaffolding), acuñado por Wood, Bruner y Ross (1976) a partir del concepto de "zona 
de desarrollo próximo" de Vygotsky (1979), hace referencia al "proceso de apoyo y control, por parte del profesor, de los aspectos de la tarea que superan las capacidades del estudiante" (López-Vargas, Hederich \& Camargo, 2012, p. 16). El andamiaje, llevado a cabo con un proceso de evaluación formativa claro, permite saber a los estudiantes qué se espera de ellos como parte de las actividades de enseñanza y aprendizaje, les proporciona la posibilidad de reorientar sus acciones sin asociarlas a la calificación, y les dirige hacia una evaluación sumativa coherente, nada sorprendente y ajustada a un esfuerzo aceptado.

\section{Contextualización del taller: Educación Universitaria}

La construcción de un andamiaje idóneo para generar aprendizaje compartido requiere de la planificación adecuada del proceso de evaluación formativa. Sin embargo, éste no tiene sentido sin el diseño, selección y presentación coherente de los instrumentos de evaluación que se vayan a utilizar. En la actualidad, los instrumentos de evaluación son definidos como "aquellos documentos o recursos que se vinculan a una actividad de evaluación, para establecer en ellos los requisitos y los aspectos a valorar, indicando con claridad los niveles de logro de cada uno" (López-Pastor \& Pérez-Pueyo 2017, p. 80). Por ello, los instrumentos, cuando se utilizan desde una perspectiva formativa, nos permiten mejorar los procesos de enseñanza y aprendizaje y "sirven para que el alumnado aprenda más (y/o corrija sus errores) y para que el profesorado aprenda a trabajar mejor (a perfeccionar su práctica docente)" (López-Pastor, 2006, p. 36). En este sentido, la aplicación en procesos triádicos de evaluación (Domínguez-Fernández et al., 2015; Pérez-Pueyo, 2017, 2018; Pérez-Pueyo \& Sobejano, 2017; Pérez-Pueyo, Casado \& Hortigüela, 2019), a través del cotejo de la autoevaluación, coevaluación y heteroevalaución llevada a cabo sobre la misma producción, permite generar un plus en cuanto al asentamiento del aprendizaje final y la valoración del mismo debido al triple enfoque que supone.

\section{Descripción del taller}

El taller pretende hacer consciente al docente de los cuatro aspectos o pasos fundamentales en la aplicación de instrumentos de evaluación en procesos formativos: (1) La necesidad de utilizar, adecuar o diseñar (si fuese el caso) el instrumento que mejor se ajuste a la actividad y producción solicitada; (2) Desarrollar un proceso de 
comprensión del instrumento por parte del alumnado, en este caso a través del andamiaje estructurado; (3) Identificar con claridad los momentos de aplicación del instrumento, los agentes implicados y el tipo de procedimiento a emplear; y (4) Llevar a cabo un proceso de evaluación triádica, si es posible, para comprobar la fiabilidad del instrumento y la justicia/coherencia de la valoración final.

- Paso 1. En este punto, dentro del "Sistema de evaluación y calificación de la asignatura", es necesario describir la actividad que se ha requerido al alumnado y que servirá de ejemplo en el taller. Actividad asociada: El alumnado, tras haber diseñado un instrumento de evaluación o calificación en cualquier ámbito de actuación de los graduados en Ciencias de la Actividad Física y el Deporte, deberá elaborar un informe de 1000 palabras cuya intención sea presentar y justificar las decisiones tomadas durante el diseño del instrumento. Comenzaremos el taller presentando el proceso de andamiaje diseñado y realizando esta actividad que clarifique al alumnado las exigencias del proceso sumativo. Éste permitirá a los asistentes comprobar las intenciones del instrumento diseñado por el docente (escala de valoración) y el orden de los aspectos a valorar en el informe que debe presentarse.

- Paso 2. Seguiremos analizando un informe y realizando un proceso de valoración del mismo con la escala de valoración elaborada ad hoc. Posteriormente cotejaremos las valoraciones de los asistentes al taller con la realizada por el docente y los alumnos que se autoevaluaron. Y analizaremos los posibles desajustes.

- Paso 3. Continuaremos explicando las razones de la elección de una escala de valoración en vez de las otras dos posibilidades de escalas descriptivas posibles como la rúbrica o la escala graduada.

- Paso 4. Y finalizaremos realizando un análisis de los pasos adecuados para la puesta en práctica en una asignatura, tras la concreción por parte del docente del instrumento de evaluación o calificación.

\section{Reflexiones finales}

El taller pretende proporcionar a los docentes un protocolo de incorporación de instrumentos de evaluación con carácter formativo, tras la elección de los más adecuados para las actividades que se pretenden llevar a cabo. Además, el poder elegir 
entre los instrumentos existentes el considerado más adecuado, dentro de las escalas que describen los niveles de logro de alumnado, facilitará la valoración final ajustada del trabajo de los estudiantes. De este modo, se pretende involucrar al alumnado en el proceso de aprendizaje a través de un adecuado andamiaje. Esto permite desarrollar procesos de evaluación triádica que faciliten el cotejo de las valoraciones de los diferentes agentes implicados, comparando la valoración de la autoevaluación y de la coevaluación con la heteroevaluación que realiza el docente,

\section{Referencias}

Biggs, J. (2005). Calidad del aprendizaje universitario. Madrid: Narcea.

Black, P. \& Williams, D. (1998). Assessment and classroom learning. Assessment in Education, 4(1), 7-71. doi: 10.1080/0969595980050102

Boud, D. (1995). Enhancing Learning Through Self Assessment. London: Routledge.

Boud, D. \& Falchikov, N. (2007). Rethinking Assessment in Higher Education. Learning for the long term. Oxon: Routledge.

Dochy, F., Segers, M., \& Dierick, S. (2002). Nuevas vías de aprendizaje y enseñanza y sus consecuencias: una era de evaluación. Revista de Docencia Universitaria, 2(2), 13-30.

Condemarín, M. \& Medina A. (2000). Evaluación de los Aprendizajes: un medio para mejorar las competencias lingüisticas y comunicativas. Chile: Ministerio de Educación.

Domínguez-Fernández, R., González-Alonso, M. I., Fernández-Fernández, R., PérezPueyo, A., Gutiérrez García, C., Alonso-Cortés Fradejas, M. D., DiezFernández, A., \& García-González, M. E. (2015). Propuesta para una doble evaluación triádica del Máster de Educación Secundaria. En A. Fidalgo Blanco, M. L. Sein-Echaluce Lacleta, \& F. J. García- Peñalvo (Coords.). La sociedad del Aprendizaje. Actas del III Congreso internacional sobre Aprendizaje Innovación y Competitividad (pp. 565-570. Madrid: Fundación General de la Universidad Politécnica de Madrid.

López-Pastor, V. M. (1999) Prácticas de evaluación en Educación Física: estudio de casos en Primaria, Secundaria y Formación del Profesorado. Valladolid: Universidad de Valladolid. 
López-Pastor, V.M. (Coord.) (2006). La Evaluación en Educación Física. Revisión de los modelos tradicionales y planteamiento de una alternativa: la evaluación formativa y compartida. Buenos Aires: Miño y Dávila

López-Pastor, V. M. (2013). Evaluación en Educación Física. Revisión internacional de la temática. Revista de Educación Física. Renovar la teoría y la práctica, 29(3), 4-13.

López-Pastor, V. M., \& Pérez-Pueyo, A. (2017). Aclaración de los términos implicados en el proceso de evaluación educativa. E, V. M. López-Pastor \& Á. PérezPueyo, A. (coords.), Evaluación formativa y compartida en Educación: experiencias de éxito en todas las etapas educativas (pp. 70-91). León: Universidad de León. Recuperado de http://buleria.unileon.es/handle/10612/5999

López-Vargas, O., Hederich, C. \& Camargo, A (2012). Logro de aprendizaje en ambientes hipermediales: andamiaje autorregulador y estilo cognitivo. Revista Latinoamericana de Psicología, 44(2), 13-26

Moreno-Olivos, T. (2007). La evaluación del aprendizaje en educación superior. El caso de la carrera de Derecho. REencuentro. Análisis de Problemas Universitarios, (48), 61-67.

Pérez-Pueyo, A. (2017). El estilo actitudinal como propuesta metodológica vinculada a la evaluación formativa. En V. M. López-Pastor \& Pérez-Pueyo (coords) Evaluación formativa y compartida en educación: experiencias de éxito en todas las etapas educativas (pp. 240-259). León: Universidad de León.

Pérez-Pueyo, A. (2018). La formación y el perfil del profesorado, reflexiones desde la teoría y la práctica. Cuadernos de Pedagogía, (489), 102-106

Pérez-Pueyo, A. \& Sobejano, M. (2017). Elaboración de instrumentos (escalas de valoración y Graduadas) para la evaluación formativa. Infancia, Educación y Aprendizaje (IEYA). 3(2), 808-814. doi: 10.22370/ieya.2017.3.2.823

Pérez-Pueyo, A., Casado, O. \& Hortigüela, D. (2019). La evaluación formativa, la autorregulación y la secuenciación de las competencias. En J. Manso \& J. Moya. Profesión y profesionalidad docente. Una acción educativa comprometida con el desarrollo humano (pp. 103-120). Madrid: Asociación Nacional de Editores de Libros y material de Enseñanza (ANELE). 
Richard, J. F. \& Godbout, P. (2000). Formative Assessment as an integral part of the teaching-learning process. Physical and Health Education Journal, 66(3), 4-9.

Villardón, L. (2006). Evaluación del aprendizaje para promover el desarrollo de competencias. Educatio siglo XXI, 24, 57-76.

Vigotsky, L. S. (1979). El desarrollo de los procesos psicológicos superiores. Barcelona: Crítica.

Wood, D., Bruner, J. \& Ross, G. (1976). The role of tutoring in problem solving. Journal of Child Psychology and Psychiatry, 17(2), 89-100. doi: 10.1111/j.14697610.1976.tb00381.x 